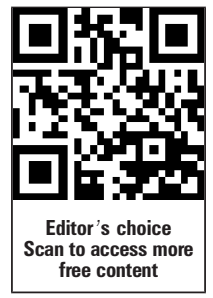

${ }^{1}$ Cancer Research UK Health Behaviour Research Centre, University College London, London, UK

${ }^{2}$ UK Centre for Tobacco Control Studies, City Hospital,

Nottingham, UK

3Primary Care Clinical Sciences, University of Birmingham, Birmingham, UK

${ }^{4}$ University College London Research Department of Clinical, Educational and Health Psychology, University College London, London, UK

\section{Correspondence to}

Emma Beard, Cancer Research UK Health Behaviour Research Centre, Department of

Epidemiology and Public Health, University College London, 1-19 Torrington Place, London, WC1E 7HB, UK; e.beard@ucl.ac.uk

Received 4 April 2011 Accepted 26 October 2011

Published Online First

1 December 2011

\title{
Association between use of nicotine replacement therapy for harm reduction and smoking cessation: a prospective study of English smokers
}

\author{
Emma Beard, ${ }_{1}^{1}$ Ann McNeill, ${ }^{2}$ Paul Aveyard, ${ }^{3}$ Jenny Fidler, ${ }^{1}$ Susan Michie, ${ }^{4}$ \\ Robert West ${ }^{1}$
}

\section{ABSTRACT}

Aims It is important to know how far smokers' attempts at using nicotine replacement therapy (NRT) for smoking 'harm reduction' (reducing harm from continued smoking) promote or undermine cessation. To contribute to that goal, this study aimed to assess whether smokers' reports of smoking reduction (SR) and the use of NRT for SR and temporary abstinence (TA) predicted subsequent attempts to quit smoking and smoking status in a population sample. It also examined whether use of NRT for SR or TA was associated with reduced cigarette consumption compared with SR without NRT and non-use of NRT for TA.

Method Data were collected from 15539 smokers involved in the Smoking Toolkit Study, a series of monthly household surveys of adults aged 16+; of whom $23 \%(n=3149)$ completed a 6-month follow-up questionnaire. At baseline, participants were asked whether they were currently using NRT for SR or TA. They were also asked for demographic information and daily cigarette consumption. At 6-month follow-up, data on attempts to quit smoking and smoking status were collected.

Results NRT use for SR and TA prospectively predicted attempts to quit smoking (OR 1.61, 95\% Cl 1.30 to 2.01 and $\mathrm{OR} 1.94,95 \% \mathrm{Cl} 1.56$ to 2.38 for SR and TA respectively) and abstinence (OR $1.51,95 \% \mathrm{Cl} 1.06$ to 2.16 and $\mathrm{OR} 2.09,95 \% \mathrm{Cl} 1.51$ to 3.34 for SR and TA respectively) at 6-months follow-up. Use of NRT for SR or TA was associated with a small reduction in cigarette consumption (two cigarettes per day) compared with SR without NRT or non-use of NRT for TA.

Conclusions The use of NRT for SR or TA appears to be positively associated with subsequent attempts to quit smoking and abstinence among smokers in England, despite very little apparent effect on daily cigarette consumption. With replication, these findings support the potential benefit of using NRT for harm reduction but primarily as a means of promoting cessation.

\section{INTRODUCTION}

In any given year, a substantial proportion of smokers do not make a quit attempt ${ }^{12}$. 'Harm reduction' has been mooted as an appropriate goal in these cases. ${ }^{3}$ We can define this as any attempt to reduce the harm, psychological or physical, from smoking without complete cessation. ${ }^{4}$ Many smokers report that they are trying to cut down the amount they smoke. ${ }^{1}$ Also, many report using nicotine replacement therapy (NRT) either as a means of smoking reduction (SR) (ie, cutting down the number of cigarettes smoked each day) or to aid temporary abstinence (TA) (ie, periods of time when one is not permitted to smoke). NRT has been licensed for use in these ways in several countries including the UK, Australia, Canada and Brazil. ${ }^{5}$ This liberalisation of the NRT licence was at least partly on the basis of data from clinical trials demonstrating that the use of NRT for SR can move smokers towards a successful quit attempt. $^{6}$

It is important to determine whether the use of NRT for SR or to aid TA can promote cessation at a population level, outside of these clinical trials. In the population there will be less structure, little or no behavioural support and NRT is not usually free of charge. In addition, different types of smokers may be involved, with clinical trials largely excluding those with chronic physical or mental conditions who appear to be the most interested in a harm reduction approach. ${ }^{7-9}$ This paper examines this issue by assessing the association between the use of NRT for SR and TA with subsequent attempts to quit smoking, the success of those quit attempts and cigarette consumption, in a general population sample of smokers in England.

We previously reported results from a crosssectional survey in which we found that the current use of NRT for SR and TA was positively associated with reports of having tried to quit in the past year. ${ }^{1}$ This suggests that use of NRT for harm reduction does not undermine attempts at cessation and may enhance it at a population level. However, it could also be that the use of NRT for harm reduction was an after effect of an unsuccessful quit attempt, ${ }^{10}$ or that the use of NRT and attempts to quit smoking are manifestations of a common underlying motivation to mitigate the harmful effects of cigarette smoking. A better test is to examine how far use of NRT for harm reduction predicts quit attempts prospectively. Finding a positive association would not prove that the use of NRT for harm reduction results in an increased likelihood of cessation but it would provide real-world evidence in support of the randomised controlled trial (RCT) findings. It would also militate strongly against the view of any negative impact of NRT for harm reduction on cessation. Our study was also able to examine separately the potential implications for subsequent cessation of using NRT for SR and TA. Although many studies have assessed the association prospectively between cessation and unaided $\mathrm{SR},{ }^{11-13}$ reporting an increased propensity to quit 
smoking among those cutting down their cigarette consumption; to our knowledge only one small study has examined the association with use of NRT for TA and SR. This failed to detect any relationship with either smoking status or attempts to quit smoking. ${ }^{14}$

To help with interpretation of the main findings of the study, we also examined how far starting or stopping the use of NRT for SR or TA was associated with changes in cigarette consumption. We previously reported from our cross-sectional study that use of NRT for SR was associated with higher cigarette consumption than for SR without $\mathrm{NRT}^{1}$ but this could have been due to heavier smokers being more likely to use NRT to aid them with reduction. The present study afforded the possibility to examine whether a change in NRT use was associated with a change in cigarette consumption in the same smokers. This will provide a clearer indication as to whether using NRT for SR is associated with more successful reduction outside of clinical trials. ${ }^{6}$

We also examined the stability of use of NRT for harm reduction. There is evidence for moderate stability of reports of attempts to reduce consumption. ${ }^{15} 16$ However, we are unaware of studies examining the stability over time of use of NRT for SR or TA. Clearly, if the use of NRT for such purposes is highly unstable, it would militate against it having any clinical benefit or impact on cessation.

Thus, the main research question addressed in this paper were:

1. Does use of NRT for SR or to aid TA predict subsequent attempts to stop and smoking status in a general population sample of smokers?

Two subsidiary questions were:

2. To what extent is starting or stopping use of NRT for SR and TA associated with changes in daily cigarette consumption?

3. What is the degree of stability over a 6-month period of use of NRT for SR or TA?

\section{METHODS \\ Study design and sampling}

The study formed part of the Smoking Toolkit Study (http:// www.smokinginengland.info), an ongoing population study designed to provide information on smoking and smoking cessation patterns among smokers and recent ex-smokers in England. Data for this paper were obtained between February 2007 and November 2010. The Smoking Toolkit Study involves monthly household surveys using a random location sampling design, with initial random selection of grouped output areas (containing 300 households), stratified by ACORN (sociodemographic) characteristics (http//www.caci.co.uk/acorn/ acornmap.asp) and region. Interviewers then choose which houses within these areas are most likely to fulfil their quotas and conduct face-to-face computer-assisted interviews with one member per household (see Fidler et al ${ }^{17}$ for further details).

\section{Measures}

Data from current smokers on demographic characteristics (gender, age and social grade) were collected. Social grade was classified as follows: $\mathrm{AB}=$ higher and intermediate professional/ managerial; $\mathrm{C} 1$ = supervisory, clerical, junior managerial/ administrative/ professional; $\mathrm{C} 2$ = skilled manual workers; $\mathrm{D}=$ semiskilled and unskilled manual workers and $\mathrm{E}=$ on state benefit, unemployed, lowest grade workers.

Participants were also asked, "Are you currently trying to cut down on how much you smoke but not currently trying to quit?" (Yes, No, Don't know). If they answered 'yes' they were asked "Which, if any, of the following are you currently using to help you cut down the amount you smoke?" (nicotine patch, nicotine gum, nicotine lozenges/tablets, nicotine inhaler, nicotine nasal spray, I don't know, none of these, other). They were also asked "Do you regularly use any of the following in situations when you are not allowed to smoke?" (nicotine patch, nicotine gum, nicotine lozenges/tablets, nicotine inhaler, nicotine nasal spray, I don't know, none of these, other).

Following the baseline survey, participants who agreed to be contacted for follow-up were sent a postal questionnaire 6 months later. Participants were asked about their smoking status, if they were reducing their smoking and if they were using NRT for SR and/or TA. Also assessed was whether an attempt to stop smoking between baseline and follow-up had occurred and cigarette consumption. Smokers were classified as being non-smokers at follow-up if they reported that they were no longer smoking and their quit attempt had started at least 4 weeks before follow-up.

\section{Analysis}

All findings are report in line with STROBE guidelines (http:// www.strobe-statement.org/index.php?id=available-checklists). Data were weighted to match the sample to the 2001 census during analysis of prevalence using a marginal weighting technique. This involves an iterative sequence of weighting adjustments whereby separate nationally representative target profiles are set (for gender, working status, prevalence of children in the household, age, social grade and region), and the process repeated until all variables match the specified targets.

Associations between the use of NRT for SR and TA at baseline with reports of attempts to stop between baseline and follow-up, and smoking status at follow-up, were assessed using multiple logistic regression, adjusting for socio-demographic and smoking characteristics. First of all, the associations of SR per se with quit attempts and smoking status were established as a prelude to the main analyses. Then, those using NRT for SR were compared with those attempting SR without NRT and those using NRT for TA were compared with all those not doing so. To assess whether use of NRT for SR or TA was associated with reduced cigarette consumption, we identified those who were smokers at both baseline and follow-up and on one occasion were using NRT and on the other occasion were not. Then, we compared the reported daily consumption between these two instances irrespective of the order in which they occurred using a paired t-test. We did this separately for use of NRT for SR and TA. For completeness, we did the same analysis for those who simply reported SR on one occasion and not the other, regardless of the use of NRT. Stability of the use of NRT for SR or TA was assessed by logistic regression to compare the odds of undertaking each activity at follow-up in those undertaking or not undertaking it at baseline.

\section{RESULTS}

Between February 2007 and November 2010, 69428 adults were surveyed; of whom, 15539 reported that they were current smokers, with an average age of 40.4 years ( $S D \pm 16.09$ ), and cigarette consumption of 13.3 cigarettes per day (SD \pm 8.43 ). Fifty-one per cent $(n=7978)$ were male with percentages in each social grade as follows: AB 15.3\% $(n=2382)$, C1 25.2\% ( $n=3915)$, C2 $24.6 \%(n=3818), D 21.9 \%$ (3408) and E 13.0\% $(n=2017)$. Sixty-eight per cent $(n=10561)$ reported smoking a cigarette within $30 \mathrm{~min}$ of wakening.

Eighty-nine per cent of smokers $(n=13917)$ agreed to be re-contacted but only $23 \%(n=3149)$ responded at 6 months 
Table 1 Characteristics of responders and non-responders

\begin{tabular}{lll}
\hline & $\begin{array}{l}\text { Respondents at } \\
\text { follow-up }(\mathbf{n = 3 1 4 9 )}\end{array}$ & $\begin{array}{l}\text { Non-respondents at } \\
\text { follow-up (n=12 390) }\end{array}$ \\
\hline Male, \% (n) & $46.2(1454)$ & $52.7(6524)^{*}$ \\
Age, mean (SD) & $46.1(15.42)$ & $38.9(15.93)^{*}$ \\
Social grade, \% (n) & & \\
$\quad$ AB & $17.8(559)$ & $14.7(1823)$ \\
C1 & $24.0(757)$ & $25.5(3158)$ \\
C2 & $23.8(748)$ & $24.8(3070)$ \\
D & $21.0(660)$ & $22.2(2747)$ \\
E & $13.4(423)$ & $12.8(1592)$ \\
Cigarettes per day, mean (SD) & $14.1(8.61)$ & $13.0(8.37)^{*}$ \\
Smoke within 30 min of & $55.8(1756)$ & $53.0(6567)^{*}$ \\
waking, \% (n) & & \\
\hline
\end{tabular}

Table entries are weighted to match the 2001 census.

* Significant difference between responders and non-responders, $\mathrm{p}<0.001$, detected using $\chi^{2}$ test for percentages and t-test for means.

after one reminder. This formed the final sample for the study. Table 1 shows the characteristics of responders and nonresponders at baseline. The differences between respondents and non-respondents were small but with the large sample size all were statistically significant. Twenty-eight per cent $(n=889)$ of respondents reported that they had attempted to quit smoking between baseline and follow-up. Nine per cent $(n=297)$ reported that they were no longer smoking at follow-up; $80 \%(n=224)$ of these had not smoked for the last 4 weeks.

Fifty-one per cent $(n=1611)$ of respondents reported at baseline that they were reducing their cigarette consumption; $25.0 \%$ of these ( $n=402 ; 12.8 \%$ of all smokers) were doing so with the aid of NRT. Thirteen per cent $(n=407)$ reported that they were using NRT for periods of TA. There was considerable overlap in the use of NRT, with $6.6 \%(n=209)$ of all smokers using NRT both for SR and TA. The high degree of overlap between use of NRT for SR and for TA made it unrealistic in the present study to dissociate the associations between these two behaviours because of very low power. Therefore, we analysed them separately. This clearly limits the extent to which conclusions can be drawn about one versus the other.

The most commonly used NRT product for SR was the nicotine patch $(n=216 ; 54 \%)$ followed by the nicotine gum $(n=133 ; 33 \%)$. This was also true for TA, with $44 \%$ reporting using the nicotine patch $(n=181)$ and $36 \%$ reporting using the nicotine gum ( $\mathrm{n}=148)$.

Table 2 shows the percentages of participants who reported (a) having attempted to stop smoking and (b) having not smoked for 4 weeks at follow-up as function of their harm reduction activities at baseline. Table 3 shows the results of the logistic regression analyses on these data. Those who reported attempting SR at baseline were more likely to report having attempted to stop smoking and less likely to be smokers at follow-up than those who did not. Those who used NRT for SR and TA were also more likely to report having attempted to stop and not to be smoking at follow-up than those attempting SR and TA without using NRT.

There was no difference in cigarette consumption when smokers changed from SR to no SR or vice versa (14.8 when not reducing vs 15.4 when reducing; $\mathrm{N}=1012$, $\mathrm{t}=1.4$, df 1996, $p=0.15$. Cigarette consumption was slightly lower when smokers were using NRT for SR than when the same smokers were attempting SR without NRT (12.7 vs 14.1, $\mathrm{N}=238, \mathrm{t}=2.02$, df $462, p=0.04)$. Similarly, use of NRT for TA was associated with lower cigarette consumption than when the same smokers were not doing this (14.7 vs 16.7, $\mathrm{N}=381, \mathrm{t}=2.30$, df 745, $\mathrm{p}=0.02$ ).

A total of 2852 reported smoking at both time points. Among this group, $72.6 \%$ of those who reported reducing their cigarette consumption at baseline continued to do so at follow-up. In contrast, only $37.2 \%$ of those using NRT for SR and $25.3 \%$ of those using NRT for TA at baseline were still using NRT for these purposes at 6 months. Table 4 shows this in more detail, with the percentage of participants attempting SR, and using NRT for SR and TA at both time points, and those starting and stopping these activities. Those reporting SR at baseline were more likely to report SR at 6-month follow-up (OR 3.3, 95\% CI 2.9 to $3.9, \mathrm{p}>0.05)$. Only a minority of those who were using NRT for SR or TA at baseline reported doing each of these at follow-up, but the odds of their using NRT for SR and TA were significantly greater than those who had not used NRT for SR or TA at baseline (OR 5.6, 95\% CI 4.0 to 7.9, p $<0.001$ vs OR 6.8, $95 \%$ CI 5.0 to $9.2, \mathrm{p}<0.001$, respectively). Thus, there was only moderate stability in these harm reduction behaviours.

\section{DISCUSSION}

The use of NRT for SR and TA was found to be positively associated with attempts to quit smoking and with abstinence at 6-month follow-up. Use of NRT for SR and TA was associated with a small reduction in daily cigarette consumption. NRT use for TA and SR was moderately stable over a 6-month period.

These findings support those of clinical trials which found that SR with NRT led to higher quit rates. ${ }^{6}$ By themselves, our

Table 2 Percentages of smokers reporting 4-week point prevalence cessation and attempts to quit smoking between baseline and 6-months follow-up as a function of smoking reduction and the use of NRT for smoking reduction and/or temporary abstinence

\begin{tabular}{|c|c|c|c|c|c|c|}
\hline & \multicolumn{2}{|c|}{ Reported SR at baseline* } & \multicolumn{2}{|c|}{$\begin{array}{l}\text { Reported NRT use for SR at } \\
\text { baseline } \dagger\end{array}$} & \multicolumn{2}{|c|}{$\begin{array}{l}\text { Reported NRT use for TA at } \\
\text { baseline } \ddagger\end{array}$} \\
\hline & Yes $(n=1611)$ & No $(n=1538)$ & Yes $(n=402)$ & No $(n=1209)$ & No $(n=407)$ & Yes $(n=2742)$ \\
\hline \multicolumn{7}{|c|}{ Quit attempt from baseline to 6 months, $\%$ (n) } \\
\hline Yes & $37.0(596)$ & $19.1(293)$ & $45.8(184)$ & $34.2(413)$ & $40.0(163)$ & $26.5(726)$ \\
\hline No & $63.0(1015)$ & $80.9(1245)$ & $54.2(218)$ & $65.8(796)$ & $60.0(244)$ & $73.5(2016)$ \\
\hline \multicolumn{7}{|c|}{ Quit smoking at 6 months, $\%(n)$} \\
\hline Yes & $9.4(152)$ & $6.0(92)$ & $11.2(45)$ & $8.9(107)$ & $12.3(50)$ & $7.1(195)$ \\
\hline No & $90.6(1459)$ & $94.0(1446)$ & $88.8(357)$ & $91.1(1102)$ & $87.7(357)$ & $92.9(2547)$ \\
\hline
\end{tabular}


Table 3 Association between smoking reduction and the use of NRT for smoking reduction and/or temporary abstinence with attempts to quit smoking and smoking status (4-week point prevalence cessation) at 6-months follow-up

\begin{tabular}{|c|c|c|c|c|c|c|}
\hline & \multicolumn{3}{|l|}{ Unadjusted } & \multicolumn{3}{|l|}{ Adjusted $\dagger$} \\
\hline & SR vs not SR $\ddagger$ & $\begin{array}{l}\text { SR with NRT vs } \\
\text { SR without NRT§ }\end{array}$ & $\begin{array}{l}\text { TA with NRT vs } \\
\text { non-NRT for TA }\end{array}$ & SR vs not SR $\ddagger$ & $\begin{array}{l}\text { SR with NRT vs } \\
\text { SR without NRT§ }\end{array}$ & $\begin{array}{l}\text { TA with NRT vs } \\
\text { non-NRT for TA }\end{array}$ \\
\hline $\begin{array}{l}\text { Quit attempt from } \\
\text { baseline to } 6 \text { months }\end{array}$ & $2.54(2.17 \text { to } 3.00)^{* * *}$ & $1.60(1.29 \text { to } 1.98)^{* * *}$ & $1.91(1.56 \text { to } 2.35)^{* * *}$ & $2.50(2.13 \text { to } 2.93)^{* * *}$ & $1.61(1.30 \text { to } 2.01)^{* * *}$ & $1.94(1.56 \text { to } 2.38)^{* * *}$ \\
\hline $\begin{array}{l}\text { Quit smoking } \\
\text { at } 6 \text { months }\end{array}$ & $1.55(1.19 \text { to } 2.01)^{* * *}$ & $1.47(1.03 \text { to } 2.10)^{*}$ & $1.98(1.44 \text { to } 2.71)^{* * *}$ & $1.49(1.14 \text { to } 1.94)^{* *}$ & $1.51(1.06 \text { to } 2.16)^{*}$ & $2.10(1.52 \text { to } 2.89)^{* * *}$ \\
\hline
\end{tabular}

${ }^{*} \mathrm{p}<0.05,{ }^{* *} \mathrm{p}<0.01,{ }^{* *} \mathrm{p}<0.001$ significant difference between groups.

†Adjusted for age, gender, social grade and time to the first cigarette of the day.

$\ddagger$ Those reporting smoking reduction compared with those reporting that they were not reducing their cigarette consumption.

$\S$ Those reporting smoking reduction with NRT versus those reducing their smoking without NRT.

qThose reporting temporary abstinence with NRT versus not using NRT for temporary abstinence.

NRT, nicotine replacement therapy; OR, odds ratio calculated from logistic regression; SR, smoking reduction; TA, temporary abstinence.

results could be interpreted in terms of use of NRT for SR and cessation both stemming from a greater sustained motivation to stop smoking. However, taken together with the findings of the clinical trials, they strengthen the view that using NRT for SR enhances the chances of subsequent quitting. The current findings also suggest that the use of NRT for TA may increase the propensity of smokers to quit. However, as has been previously reported, ${ }^{1}$ there was considerable overlap between the use of NRT for SR and for TA, which meant it was not possible to determine the specific association between these different uses of NRT and outcome variables.

Our findings lend further support to the recommendation that NRT be used for SR or TA, but cannot rule out the possibility that promoting use of NRT in this way might have other adverse consequences that might reduce population quit rates. Thus is it conceivable that those who would previously have made a quit attempt would instead try to reduce, and this effect may be large enough to more than offset any gain in quitting resulting from such a reduction. This issue could be addressed by observing population-level quit rates before and after the introduction of marketing licences allowing the use of NRT for SR. Even then, there are clearly many factors that could come into play to affect those rates, so disentangling the effect of those interested in abrupt cessation switching to SR would be difficult. It may be noted that there is no evidence to date that marketing of NRT for SR or changes to tobacco control policy have made any difference to the rate at which it has been used for this purpose, with little change in prevalence over the past 5-6 years. ${ }^{1} 18$ This may be because the marketing activity has been very limited. It remains to be seen what will happen if and when the manufacturers engage in more vigorous marketing and use of NRT for SR increases.

Table 4 Percentages of smokers reporting smoking reduction and the use of NRT for smoking reduction and/or temporary abstinence at baseline and follow-up

\begin{tabular}{|c|c|c|c|}
\hline & $\begin{array}{l}\text { Smoking } \\
\text { reduction }\end{array}$ & $\begin{array}{l}\text { Using NRT } \\
\text { for smoking } \\
\text { reduction }\end{array}$ & $\begin{array}{l}\text { Using NRT } \\
\text { for temporary } \\
\text { abstinence }\end{array}$ \\
\hline At baseline, $n$ & 1433 & 261 & 352 \\
\hline Continuing at follow-up, \% (n) & $72.6(1040)$ & $37.2(97)$ & $25.3(89)$ \\
\hline Stopping at follow-up, \% (n) & $27.4(393)$ & $62.8(164)$ & 74.7 (263) \\
\hline Not at baseline, $n$ & 1419 & 779 & 2500 \\
\hline Not at follow-up, \% (n) & $56.4(800)$ & $90.5(705)$ & $95.3(2382)$ \\
\hline Starting at follow-up, \% (n) & $43.6(619)$ & $9.5(74)$ & $4.7(118)$ \\
\hline
\end{tabular}

Table entries are weighted to match the 2001 census. Table entries are based on reports of smoking at baseline and follow-up $(n=2852)$; those reporting that they were no longer smoking at follow-up were excluded.

NRT, nicotine replacement therapy.
The moderate stability of use of NRT for SR and TA is in line with previous research, suggesting that only $2.3 \%$ of purchases of NRT lead to continuous monthly purchases. ${ }^{19} 20$ This may be partially explained by the cost of purchasing NRT concurrently with cigarettes which may lead to smokers not using as much NRT or terminating its use sooner. However, this cannot be the only explanation as many smokers continue to smoke despite rises in the cost of cigarettes. Smokers using NRT for TA as a result of smoke-free laws may also find the current products ineffective. ${ }^{21}$ In line with this, previous studies have found that few smokers report that NRT helps them to resist urges to smoke in situations where smoking is not possible. ${ }^{22}$ Further research is needed to explore the issue of stability further. This study only looked at two points in time. It will be important to ask smokers about how long they have been using NRT in particular ways and indeed to chart the trajectory of their NRT use and cessation attempts over an extended time period.

Although we found that a change in NRT-use status was associated with a change in cigarette consumption, this change was small. These findings are in line with previous populationbased data ${ }^{1}$ but are in contrast to the significant reductions reported in the clinical trials. ${ }^{6}$ Of course, it is possible that NRT use was having an effect not on consumption but on the amount of nicotine ingested per cigarette. There is evidence to support this view. ${ }^{23}$ Use of NRT in the previous clinical trials was also relatively high compared with usage of NRT while concurrently smoking at a population level, ${ }^{24}$ and this may influence whether reliable reductions in cigarette consumption occur. This is an important area for future research.

This study had several limitations. First, there may be error or bias in the measurement of the key variables. For example, it has been found that smokers appear to forget failed quit attempts relatively quickly, especially if they did not last more than a few days. ${ }^{25}$ However, there is no reason to assume that the rate of forgetting would differ between reducers and non-reducers, and therefore, this would not have created spurious associations. Second, the follow-up rate was low. However, differences between responders and non-responders were small, and it is not clear how self-selection bias could account for the effects observed. Nevertheless, it will be important to continue to gather data on this topic using other population data sets as these become available. Third, we did not measure the amount of NRT used. It could be that this played a significant role in any association with cessation or reduced cigarette consumption. Finally, we did not have information on use of NRT and smoking behaviour between the baseline and 6-month surveys; it is possible that smokers started and stopped using NRT numerous times during this period. Further research on the topic of 


\section{What this paper adds}

Clinical trials have demonstrated the efficacy of the use of nicotine replacement therapy for harm reduction purposes, reporting that the concurrent use of nicotine replacement therapy and cigarettes results in significant reductions in cigarette consumption and increases the propensity of smokers to quit. It is important to determine whether similar findings appear at a population level, where the provision of nicotine replacement therapy is not generally free of charge and little behavioural support is provided. Although cross-sectional studies have established higher odds of attempts to quit smoking among those spontaneously using nicotine replacement therapy for harm reduction purposes, this could be for a number of reasons: the use of nicotine replacement therapy may be an after effect of a failed quit attempt or the use of nicotine replacement therapy may increase smokers motivation to quit. The current study aimed to resolve this issue by collecting data prospectively on smokers who were using nicotine replacement therapy for smoking reduction and/or during periods of temporary abstinence. It was found that the use of nicotine replacement therapy for such purposes was positively associated both with attempts to stop smoking and 4-week point prevalence cessation.

stability would need to adopt long-term repeated measures of NRT use.

\section{CONCLUSIONS}

Although the use of NRT for SR and TA was associated with only small reductions in cigarette consumption, it was predictive of attempts to stop smoking and abstinence 6 months later. This supports the findings of RCTs that NRT use whilst smoking may help promote cessation.

Funding This study was funded by the English Department of Health, Cancer Research UK, Pfizer, GlaxoSmithKlein and Johnson and Johnson, who had no involvement in the design of the study, the analysis or interpretation of the data, the writing of the report or the decision to submit the paper for publication.

Competing interests PA has undertaken consultancy work on smoking cessation for Celtic (Xenova), Pfizer and McNeil. EB has received conference funding from Pfizer. RW undertakes research and consultancy and receives fees for speaking from companies that develop and manufacture smoking cessation medications. He also has a share of a patent for a novel nicotine delivery device.

Patient consent Obtained.

Ethics approval Ethics approval was provided by UCL.

Contributors All authors contribute to the manuscript. EB and RW designed the study and wrote the first draft. JF, AM, PA and SM revised the manuscript.

Provenance and peer review Not commissioned; externally peer reviewed.

\section{REFERENCES}

1. Beard $\mathbf{E}, \mathbf{M c N e i l l} A$, Aveyard $P$, et al. Use of nicotine replacement therapy for smoking reduction and during enforced temporary abstinence: a national survey of English smokers. Addiction 2011;106:197-204.

2. Hyland A, Borland R, Li Q, et al. Individual-level predictors of cessation behaviours among participants in the International Tobacco Control (ITC) Four Country Survey. Tob Control 2006;15:83-94.

3. Stratton K, Shetty P, Wallace R, et al. Clearing the smoke: the science base for tobacco harm reduction: an executive summary. Tob Control 2001;10:189-95.

4. West R, Beard E, Michie S, et al. A taxonomy of harm reduction for use in tobacco control. Addiction. In press

5. Medicines and Healthcare Products Regulatory Agency. MHRA Public Assessment Report: The Use of Nicotine Replacement Therapy to Reduce Harm in Smokers. 2010. http://www.mhra.gov.uk/Safetyinformation/

Safetywarningsalertsandrecalls/Safetywarningsandmessagesformedicines/CON068572

6. Moore D, Aveyard P, Wang D, et al. A systematic review and meta-analysis of the efficacy and safety of nicotine assisted reduction to stop smoking programmes. BMJ 2009;338:1-9.

7. Moeller-Saxone K. Cigarette smoking and interest in quitting among consumers at a Psychiatric Disability Rehabilitation and Support Service in Victoria. Aust N Z J Public Health 2008;32:479-81.

8. McDermott L, Dobson A, Owen N. Smoking reduction and cessation among young adult women: a 7 year prospective analysis. Nicotine Tob Res 2008;10:1457-66.

9. Berg CJ, Thomas JL, Guo H, et al. Predictors of smoking reduction among Blacks. Nicotine Tob Res 2010;12:423-31.

10. Yong HH, Borland R, Hyland A, et al. How does a failed quit attempt among regular smokers affect their cigarette consumption? Findings from the International Tobacco Four Country Survey (ITC-4). Nicotine Tob Res 2008;10:897-905.

11. Falba T, Jofre-Bonet $\mathrm{M}$, Busch $\mathrm{S}$, et al. Reduction of quantity smoked predicts future cessation among older smokers. Addiction 2004:99:93-102.

12. Farkas AJ. When does cigarette fading increase the likelihood of future cessation? Ann Behav Med 1999;21:71-6.

13. Hyland A, Levy DT, Rezaishiraz $\mathrm{H}$, et al. Reduction in amount smoked predicts future cessation. Psychol Addict Behav 2005;19:221-5.

14. Levy DE, Thorndike AN, Biener L, et al. Use of nicotine replacement therapy to reduce or delay smoking but not to quit: prevalence and association with subsequent cessation efforts. Tob Control 2007;16:384-9.

15. West R, McEwen A, Bolling K, et al. Smoking cessation and smoking patterns in the general population: a 1-year-follow-up. Addiction 2001;96:891-902.

16. Meyer C, Rumpf JJ, Schumann A, et al. Intentionally reduced smoking among untreated general population smokers: prevalence, stability, prediction of smoking behaviour change and differences between subjects choosing either reduction or abstinence. Addiction 2003;98:1101-10.

17. Fidler J, Shahab L, West 0, et al. 'The Smoking Toolkit Study': a national study of smoking and smoking cessation in England. BMC Public Health 2011;11:479.

18. Shahab L, Cummings KM, Hammond D, et al. The impact of changing nicotine replacement therapy licensing laws in the United Kingdom: findings from the International Tobacco Control Four Country Survey. Addiction 2009;104:1420-7.

19. Shiffman S, Hughes JR, Pillitteri J, et al. Persistent use of nicotine replacement therapy: an analysis of actual purchase patterns in a population based sample. Tob Control 2003;12:310-16.

20. Shiffman S, Hughes S, Marino JR, et al. Patterns of over the counter nicotine gum use: persistent use and concurrent smoking. Addiction 2003;98:1747-53.

21. Etter JF, Le Houezec J, Landfeldt B. Impact of messages on concomitant use of nicotine replacement therapy and cigarettes: a randomised trial on the internet. Addiction 2003;98:941-50.

22. Etter JF, Perneger TV. Attitudes toward nicotine replacement therapy in smokers and ex-smokers in the general public. Clin Pharmacol Ther 2001;69:175-83.

23. Beard $\mathbf{E}$, Fidler J, West R. Is use of nicotine replacement therapy while continuing to smoke associated with increased nicotine intake? Psychopharmacology (Berl). Published Online First: 26 May 2011. doi:10.1007/s00213-011-2359-4.

24. Shiffman S, Rolf CN, Hellebusch SJ, et al. Real-world efficacy of prescription and over-the-counter nicotine replacement therapy. Addiction 2002;97:505-16.

25. Berg CJ, An LC, Kirch M, et al. Failure to report attempts to quit smoking. Addict Behav 2010;35:900-4. 